\title{
Elevated plasma copeptin levels identify the presence and severity of non-alcoholic fatty liver disease in obesity
}

Ilaria Barchetta', Sofia Enhörning ${ }^{2}$, Flavia Agata Cimini ${ }^{1}$, Danila Capoccia ${ }^{1}$, Caterina Chiappetta ${ }^{1}$, Claudio Di Cristofano ${ }^{3}$, Gianfranco Silecchia ${ }^{3}$, Frida Leonetti ${ }^{1}$, Olle Melander ${ }^{2^{*}}$ and Maria Gisella Cavallo ${ }^{{ }^{*}}$

\begin{abstract}
Introduction: Copeptin is the stable surrogate marker of vasopressin (VP), which is released in response to elevated plasma osmolality or low blood pressure. Elevated plasma copeptin levels are associated with higher risk of insulin resistance-related disorders, such as type 2 diabetes (T2DM), metabolic syndrome (MS), and cardiovascular disease, and experimental reduction of circulating VP levels is shown to significantly decrease hepatic fat content in obese rats, independently from body adiposity. However, the association between copeptin and non-alcoholic fatty liver disease and steatohepatitis (NAFLD/NASH) in humans has not been explored yet. The aim of this study was to explore the relationship between plasma copeptin and the presence/severity of NAFLD/NASH.
\end{abstract}

Methods: For this study, we recruited 60 obese patients candidate to bariatric surgery for clinical purposes in which intraoperative liver biopsies were performed for diagnosing NAFLD/NASH. Circulating copeptin levels were also assessed in 60 age- and sex-comparable non-obese individuals without NAFLD at liver ultrasonography. Plasma copeptin was measured by sandwich immunoluminometric assay (Thermo Fisher Scientific).

Results: Obese patients with biopsy-proven NAFLD (53\%) had significantly higher copeptin levels than both obese individuals without NAFLD and non-obese subjects (ob/NAFLD+9.5 \pm 4.9; ob/NAFLD- 6.4 \pm 2.6; and non-ob/NAFLD$7.4 \pm 5.1 \mathrm{pmol} / \mathrm{L} ; p=0.004$ and $p=0.01$ respectively). Plasma copeptin concentration positively correlated with hepatic macro- and micro-vesicular steatosis $(r=0.36, p=0.026 ; r=0.31, p=0.05)$, lobular inflammation $(r=0.37, p=0.024)$ and significantly increased throughout degrees of NASH severity, as expressed as absence, borderline, and overt NASH at the liver biopsy $(r=0.35, p=0.01)$. Greater circulating copeptin predicted the presence of NASH with $\mathrm{OR}=1.73$ (95\% $\mathrm{Cl}=1.02-2.93)$ after multivariate adjustment for age, sex, renal function and presence of T2DM and MS components.

Conclusions: Increased plasma copeptin is independently associated with the presence and severity of NAFLD and NASH, pointing to a novel mechanism behind human fatty liver disease potentially modifiable by pharmacological treatment and lifestyle intervention.

Keywords: Vasopressin, Copeptin, Antidiuretic hormone, Fatty liver, NAFLD, NASH, Metabolic syndrome, Obesity

\footnotetext{
* Correspondence: olle.melander@med.lu.se; gisella.cavallo@uniroma1.it

2Department of Clinical Sciences, Lund University, Malmoe, Sweden

1 Department of Experimental Medicine, Policlinico Umberto I, Sapienza

University of Rome, Rome, Italy

Full list of author information is available at the end of the article
}

(c) The Author(s). 2019 Open Access This article is distributed under the terms of the Creative Commons Attribution 4.0 International License (http://creativecommons.org/licenses/by/4.0/), which permits unrestricted use, distribution, and reproduction in any medium, provided you give appropriate credit to the original author(s) and the source, provide a link to the Creative Commons license, and indicate if changes were made. The Creative Commons Public Domain Dedication waiver (http://creativecommons.org/publicdomain/zero/1.0/) applies to the data made available in this article, unless otherwise stated. 


\section{Background}

Vasopressin (VP) is a hormone secreted by the pituitary gland in response to increased plasma osmolality, low plasma volume, and low blood pressure. Copeptin is a cleavage product of the C-terminal part of the VP precursor which correlates well with plasma VP concentrations and which is easier to measure reliably $[1,2]$. Thus, copeptin is nowadays considered the circulating surrogate biomarker of VP [2].

Beside its role in inducing reactive vasoconstriction and inhibiting diuresis and, thus, controlling blood volume [1, 2], VP exerts major effects on glucose and lipid metabolism by stimulating the hepatic glycogenolysis [3], gluconeogenesis [4], and fat production [5] and by modulating the release of insulin and glucagon from the pancreatic Langerhans' islets [6].

In humans, elevated circulating copeptin levels have been independently associated with increased risk of type 2 diabetes mellitus (T2DM), cardiovascular morbidity and mortality [7-14], and clinical signatures of metabolic syndrome (MS), such as hyperinsulinemia [7], visceral fat deposition, systemic hypertension, high triglycerides, and impaired glucose regulation, independently from obesity $[15,16]$. Of note, obese rats with elevated VP develop glucose intolerance, whereas blocking of the VP 1a receptors (V1aR) improves glucose tolerance [17].

Among metabolic disorders, non-alcoholic fatty liver disease (NAFLD) has gained special attention in the last decade since it represents the most common chronic liver disease worldwide with an estimated prevalence around $25 \%$ in the general population and up to $80 \%$ in obese individuals [18-20]. NAFLD is an established risk factor for all-cause and cardiovascular mortality [21]. However, mechanisms behind NAFLD development and progression to steatohepatitis (NASH) have not been fully unraveled, and no specific therapy has been identified yet [22].

VP exerts a direct influence on the hepatic fat metabolism by stimulating the production of triglycerides in rat hepatocytes [5]; accordingly, mice lacking expression of the VP receptor 1a have low triglycerides compared to wild type [23]. Indeed, obese rats with water-induced reduction of circulating VP levels have significantly decreased hepatic fat content compared with control obese rats, independently from changes in body adiposity [17]. In humans, clinical observations show a relationship between elevated plasma copeptin levels and the presence of hepatic cirrhosis, likely reflecting underlying circulatory dysfunction [24] suggesting a role of copeptin as a prognostic marker of liver disease [25].

Even though epidemiological and experimental evidence supports a direct involvement of VP in insulin resistance-associated disorders, to date nothing is known about the role of circulating copeptin in human NAFLD and NASH. Therefore, the aims of this study are to investigate the association between plasma copeptin and the presence and severity of NAFLD/NASH and to analyze clinical correlates of increased copeptin levels.

\section{Methods \\ Study population}

For this cross-sectional study, we recruited 60 consecutive obese subjects referring to our outpatient clinics at the Sapienza University of Rome, Italy, who were all candidates to bariatric surgery as for clinical indication, and an additional study cohort of 60 non-obese controls with comparable age and sex, who were not affected by metabolic syndrome (MS), as defined by the ATP III criteria [26], at the clinical evaluations performed in the same setting.

Eligible for this study were all the participants who had met the following inclusion criteria: male or female individuals aged between 20 and 65 years; no history of excessive alcohol drinking (considered as an average daily consumption of alcohol $>30 \mathrm{~g} /$ day in men and $>20 \mathrm{~g} /$ day in women); negative tests for the presence of hepatitis $\mathrm{B}$ surface antigen and antibody to hepatitis $C$ virus; absence of history of cirrhosis and other causes of liver diseases (hemochromatosis, autoimmune hepatitis, Wilson's disease); and no treatment with drugs known to cause liver steatosis (e.g., corticosteroids, estrogens, methotrexate, tetracycline, calcium channel blockers, or amiodarone). Additional exclusion criteria for subjects belonging to the control group were diagnosis of obesity defined as $\mathrm{BMI} \geq$ $30 \mathrm{~kg} / \mathrm{m}^{2}$, presence of MS [26], impaired glucose tolerance or T2DM, as defined by the American Diabetes Association 2009 criteria [27] and detection of hepatic steatosis at the abdomen ultrasonography assessment [28].

All the study participants underwent medical history collection, clinical work-up, and laboratory tests. Weight and height were measured with light clothes and without shoes, and the body mass index (BMI, $\mathrm{kg} / \mathrm{m}^{2}$ ) was calculated. Waist circumference $(\mathrm{cm})$ was measured midway between the 12th rib and the iliac crest. Systemic systolic (SBP) and diastolic (DBP) blood pressure were measured after $5 \mathrm{~min}$ resting; three measurements were taken, and the average of the second and third measurements was recorded and used in the analyses.

\section{Laboratory tests}

Venous blood sampling after 12-h fasting was performed in all the study participants for routine analyses and metabolic characterization. Total cholesterol (mg/dL), high-density lipoprotein cholesterol (HDL, mg/dL), triglycerides $(\mathrm{mg} / \mathrm{dL})$, aspartate aminotransferase (AST, IU/L), alanine aminotransferase (ALT, IU/L), gamma-glutamyl transpeptidase (GGT, mg/dL), fasting blood glucose (FBG, $\mathrm{mg} / \mathrm{dL})$, uric acid $(\mathrm{mg} / \mathrm{dL})$, and creatinine $(\mathrm{mg} / \mathrm{dL})$ were measured by centralized standard methods. Fasting insulin (FBI, $\mu \mathrm{IU} / \mathrm{mL}$ ) was measured by radioimmunoassay 
Table 1 Clinical and biochemical characteristic of non-obese and obese study participants according to the presence of NAFLD

\begin{tabular}{|c|c|c|c|c|c|}
\hline & $\begin{array}{l}\text { Non-obese individuals } \\
(n=60)\end{array}$ & $\begin{array}{l}\text { Obese no NAFLD } \\
(n=28)\end{array}$ & $\begin{array}{l}\text { Obese NAFLD } \\
(n=32)\end{array}$ & $\begin{array}{l}p \text { value } \\
\text { ANOVA }\end{array}$ & $\begin{array}{l}p \text { value } \\
\text { Bonferroni post hoc test }\end{array}$ \\
\hline Age (years) & $46.3 \pm 11.6$ & $40.5 \pm 12$ & $43.2 \pm 9.4$ & 0.71 & $\begin{array}{l}0.97 \wedge \\
0.90^{*} \\
0.70^{\circ}\end{array}$ \\
\hline $\operatorname{Sex}(M / F)$ & $60 \%$ & $61.5 \%$ & $32 \%$ & $0.12^{\#}$ & $\begin{array}{l}0.14 \wedge^{\prime \prime} \\
0.99^{* »} \\
0.59^{\circ »}\end{array}$ \\
\hline BMI $\left(\mathrm{kg} / \mathrm{m}^{2}\right)$ & $24.3 \pm 3.2$ & $43.5 \pm 6.3$ & $41.8 \pm 4.3$ & $<0.001$ & $\begin{array}{l}0.20 \wedge \\
<0.001^{\S}\end{array}$ \\
\hline Waist circumference $(\mathrm{cm})$ & $83.9 \pm 13$ & $129.4 \pm 16.9$ & $128 \pm 7.7$ & $<0.001$ & $\begin{array}{l}0.94 \wedge \\
<0.001^{\S}\end{array}$ \\
\hline $\mathrm{SBP}(\mathrm{mmHg})$ & $115.5 \pm 20.4$ & $133 \pm 8.4$ & $124.4 \pm 7.7$ & 0.02 & $\begin{array}{l}0.9 \wedge^{\wedge} \\
0.05^{*} \\
0.06^{\circ}\end{array}$ \\
\hline $\mathrm{DBP}(\mathrm{mmHg})$ & $75.8 \pm 8.1$ & $85 \pm 8.7$ & $85.9 \pm 22.1$ & 0.009 & $\begin{array}{l}0.98 \wedge \\
0.10^{*} \\
0.02^{\circ}\end{array}$ \\
\hline Serum creatinine (mg/dL) & $0.74 \pm 0.15$ & $0.70 \pm 0.1$ & $0.82 \pm 0.16$ & 0.03 & $\begin{array}{l}0.04 \wedge \\
0.68^{*} \\
0.09^{\circ}\end{array}$ \\
\hline Total cholesterol (mg/dL) & $199 \pm 35.1$ & $213 \pm 140.4$ & $171 \pm 126$ & 0.18 & $\begin{array}{l}0.16^{\wedge} \\
0.28^{*} \\
0.81^{\circ}\end{array}$ \\
\hline $\mathrm{HDL}-\mathrm{C}(\mathrm{mg} / \mathrm{dL})$ & $56.1 \pm 14.1$ & $52 \pm 8.8$ & $46.7 \pm 10.2$ & 0.037 & $\begin{array}{l}0.82^{\wedge} \\
0.46^{*} \\
0.03^{\circ}\end{array}$ \\
\hline LDL-C (mg/dL) & $118.9 \pm 34.8$ & $141.9 \pm 26.6$ & $121.1 \pm 22.3$ & 0.35 & $\begin{array}{l}0.33^{\wedge} \\
0.39^{*} \\
0.96^{\circ}\end{array}$ \\
\hline Triglycerides (mg/dL) & $77.5(66-137.5)$ & $101(73.7-121.5)$ & $136(117.2-164)$ & 0.09 & $\begin{array}{l}0.59 \wedge \\
0.14^{*} \\
0.48^{\circ}\end{array}$ \\
\hline Uric acid (mg/dL) & $4.3 \pm 1.2$ & $4.9 \pm 0.6$ & $5.9 \pm 1.5$ & 0.02 & $\begin{array}{l}0.02^{\wedge} \\
0.58^{*} \\
0.02^{\circ}\end{array}$ \\
\hline AST (IU/L) & $18(15-21.25)$ & $20.5(17.8-25.3)$ & $23(17-30)$ & 0.004 & $\begin{array}{l}0.52^{\wedge} \\
0.38^{*} \\
0.003^{\circ}\end{array}$ \\
\hline ALT (IU/L) & $17(13-24.25)$ & $20.5(15.8-27.3)$ & $32(18.5-47.5)$ & $<0.001$ & $\begin{array}{l}0.02^{\wedge} \\
0.79^{*} \\
<0.001^{\circ}\end{array}$ \\
\hline $\mathrm{FBG}(\mathrm{mg} / \mathrm{dL})$ & $90.5(82-95.25)$ & $95(92-101.5)$ & $99(87-125)$ & 0.02 & $\begin{array}{l}0.75^{\wedge} \\
0.09^{*} \\
0.02^{\circ}\end{array}$ \\
\hline $\mathrm{FBI}(\mu \mathrm{IU} / \mathrm{mL})$ & $10.3(1.6-17.8)$ & $9(6.3-15.5)$ & $11.5(10.5-14)$ & 0.66 & $\begin{array}{l}0.85^{\wedge} \\
0.91^{*} \\
0.64\end{array}$ \\
\hline HOMA-IR & $2.9(2.1-4.8)$ & $2(1.5-3.6)$ & $2.9(2.4-3.8)$ & 0.95 & $\begin{array}{l}0.94 \wedge \\
0.99^{*} \\
0.94^{\circ}\end{array}$ \\
\hline НОМА- $\beta \%$ & $139.3(101.2-336)$ & $111.7(68.1-180.4)$ & 133.5 (67.4-216.7) & 0.15 & $\begin{array}{l}0.85^{\wedge} \\
0.21^{*} \\
0.26^{\circ}\end{array}$ \\
\hline MS (\%) & $0 \%$ & $46 \%$ & $81 \%$ & $<0.001^{\#}$ & $\begin{array}{l}0.004 \wedge^{\prime \prime} \\
<0.001^{\S »}\end{array}$ \\
\hline T2DM (\%) & $0 \%$ & $8 \%$ & $20 \%$ & $0.01^{\#}$ & $0.19 \wedge^{\prime \prime}$ \\
\hline Treated with metformin & & $100 \%$ & $70 \%$ & & $\begin{array}{l}0.04^{* \prime \prime} \\
0.01^{\circ »}\end{array}$ \\
\hline
\end{tabular}


Table 1 Clinical and biochemical characteristic of non-obese and obese study participants according to the presence of NAFLD (Continued)

\begin{tabular}{|c|c|c|c|c|c|}
\hline & $\begin{array}{l}\text { Non-obese individuals } \\
(n=60)\end{array}$ & $\begin{array}{l}\text { Obese no NAFLD } \\
(n=28)\end{array}$ & $\begin{array}{l}\text { Obese NAFLD } \\
(n=32)\end{array}$ & $\begin{array}{l}p \text { value } \\
\text { ANOVA }\end{array}$ & $\begin{array}{l}p \text { value } \\
\text { Bonferroni post hoc test }\end{array}$ \\
\hline Treated with glinides & & $0 \%$ & $14 \%$ & & \\
\hline Treated with insulin & & $0 \%$ & $28 \%$ & & \\
\hline Use of antihypertensive agents (\%) & $0 \%$ & $36 \%$ & $81 \%$ & $<0.001^{\#}$ & $\begin{array}{l}0.003 \wedge^{\prime \prime} \\
<0.001^{\text {§” }}\end{array}$ \\
\hline Use of statins (\%) & $0 \%$ & $30 \%$ & $69 \%$ & $<0.001^{\#}$ & $\begin{array}{l}0.003 \wedge^{\prime \prime} \\
<0.001^{\text {§” }}\end{array}$ \\
\hline Copeptin (pmol/L) & $5.4(4-9.45)$ & $6.1(4.3-8.6)$ & $8.6(5.4-11.9)$ & 0.037 & $\begin{array}{l}0.019 \wedge \\
0.69^{*} \\
0.022^{\circ}\end{array}$ \\
\hline
\end{tabular}

ANOVA test. "Kruskall-Wallis test. Comparison between the following: ${ }^{\S}$ non-obese individuals vs obese +/- NAFLD; $\wedge$ obese NAFLD vs obese no NAFLD; *non-obese individuals vs obese no NAFLD; ${ }^{\circ}$ non-obese individuals vs obese NAFLD. " $X^{2}$ test for comparison between two groups. Data are expressed as a percentage, mean $\pm \mathrm{SD}$, and/or median (interquartile range), as appropriate

Abbreviations: SBP systolic blood pressure, DBP diastolic blood pressure, $H D L-C$ high-density lipoprotein cholesterol, $L D L-C$ low-density lipoprotein, $A S T$ aspartate aminotransferase, ALT alanine aminotransferase, FBG fasting blood glucose, FBI fasting blood insulin, HOMA-IR HOmeostasis Model Assessment of insulin resistance, HOMA- $\beta \%$ HOmeostasis Model Assessment of insulin secretion, MS metabolic syndrome, T2DM type 2 diabetes mellitus

(ADVIA Insulin Ready Pack 100; Bayer Diagnostics, Milan, Italy), with intra- and inter-assay coefficients of variation < $5 \%$, by an experienced lab technician, at Sapienza University, Rome, Italy. Low-density lipoprotein (LDL) cholesterol value was obtained using the Friedewald formula; the homeostasis model assessment of insulin resistance (HOMA-IR) and insulin secretion (HOMA- $\beta \%$ ) was calculated as previously described [29].

Fasting copeptin was measured in plasma samples frozen immediately after separation and stored at $-80^{\circ} \mathrm{C}$ for some weeks, using a KRYPTOR Compact Plus device and commercially available chemiluminescence sandwich immunoassay copeptin ProAVP kit with coated tubes (Thermo Scientific BRAHMS Copeptin proAVP KRYPTOR).

\section{Liver histology}

Obese patients underwent intraoperative liver biopsy during surgery for sleeve gastrectomy; all the procedures were conducted in accordance with recommendations by the American Association for the Study of Liver Diseases [30]. A single pathologist blinded to patients' medical history and biochemistry performed the overall histological evaluations. A minimum biopsy length of $15 \mathrm{~mm}$ or the presence of 10 complete portal tracts was required [31] for clinical diagnosis. Liver fragments were fixed in buffered formalin for $2-4 \mathrm{~h}$ and embedded in paraffin; sections were cut and stained with hematoxylin and eosin and Masson's trichrome stains. Liver biopsy samples were classified based on the presence of NASH by Brunt definition [32] and graded according to the NAFLD activity score (NAS) [33]; fibrosis was quantified on the basis of the NASH Clinical Research Network Scoring System Definition [33].

\section{Liver ultrasound assessment}

Study participants belonging to the control group underwent abdomen ultrasound (US) evaluation performed by the same operator blinded for patients' identity and blood tests using an Esaote Medica instrument with a convex $3.5 \mathrm{MHz}$ probe. The presence of fatty liver was defined based on the criteria by Saverymuttu et al. [28] which take into account abnormally intense, high-level echoes arising from the hepatic parenchyma, liver-kidney difference in echo amplitude, echo penetration into the deep portion of the liver, and clarity of liver blood vessel structure.

\section{Statistics}

As far as we know, this is the first study investigating plasma copeptin levels in relation to the presence of NAFLD. Thus, for confirming the statistical power of our findings, we performed a post hoc sample size calculation on the basis of mean \pm standard deviation (SD) copeptin levels in obese subjects with and without biopsy-proven NAFLD which showed that 27 subjects in each group would have been sufficient to reach the statistical significance with power $=80 \%$ and $\alpha$ error $=0.05$.

All the statistic procedures have been performed using SPSS version 23. Values are shown as mean \pm SD, median (interquartile range), or percentage, as appropriate. In this study population, plasma copeptin, triglycerides, FBG, AST, ALT, GGT, FBI, HOMA-IR, and HOMA- $\beta \%$ had skewed distribution and were log-transformed before the analyses. Mean values between two independent groups were compared by Student's $T$ test for continuous variables and by $\chi^{2}$ test for categorical parameters; comparisons between more than two subgroups were performed by the Bonferroni-adjusted ANOVA test. Bivariate correlations were explored by Pearson's (continuous variables) or Spearman's (categorical variables) coefficients. In order to identify determinants of NASH (yes/no, dependent variable) in our study population, we built multivariate logistic regression models including 
age, sex, T2DM, and components of MS, as expressed as either the numbers of MS components-ranging from 0 to 5-or entering each metabolic parameter as a continuous variable in a conditional forward logistic regression. Moreover, the predictive value of plasma copeptin for NASH identification was estimated by the area under receiver operating characteristic curve (AUROC), with a 95\% confidence interval (CI).

A two-tailed $p$ value $<0.05$ was considered statistically significant, with a $95 \%$ confidence interval.

The study protocol was reviewed and approved by the local Ethics Committee at the Sapienza University of Rome, Italy, and conducted in conformance with the Helsinki Declaration. Informed written consent was obtained from all patients before all the study procedures.

\section{Results}

Obese patients with biopsy-proven NAFLD (obese+/NAFLD+, 53\%) had significantly higher copeptin levels than obese individuals without NAFLD (obese+/NAFLD-) (obese $+/ \mathrm{NAFLD}+9.5 \pm 4.9 \mathrm{pmol} / \mathrm{L}$ vs obese+/NAFLD $-6.4 \pm 2.6$ $\mathrm{pmol} / \mathrm{L}, p=0.004)$. Moreover, copeptin levels were also greater in obese+/NAFLD+ individuals when compared with non-obese subjects without MS and NAFLD (obese-/NAFLD-, mean \pm SD copeptin $7.4 \pm 5.1 \mathrm{pmol} / \mathrm{L}$; $p=0.01)$.

The presence of significantly greater copeptin levels in obese+/NAFLD+ than both obese+/NAFLD- and obese -/NAFLD- was confirmed by the post hoc adjusted ANOVA test (model $\mathrm{F}=4.09, p=0.019 ; p=0.022$ and $p=0.034$ ), whereas no statistically significant difference was observed between plasma copeptin in obese individuals without NAFLD and non-obese control subjects (Table 1).

When comparing plasma copeptin levels between obese +/NAFLD+ and obese+/NAFLD- patients in relation to the presence of MS, the finding of higher copeptin in presence of NAFL - both NAFLD and NASH - was confirmed in the MS group $(n=42$; NAFLD- $7.1 \pm 2.7$ vs NAFLD+ $10 \pm 5.2 \mathrm{pmol} / \mathrm{L}, p=0.024$; NASH $-7.7 \pm 3.7$ vs NASH + $12.2 \pm 5.7 \mathrm{pmol} / \mathrm{L}, p=0.007$ ), and slightly confirmed in the significantly smaller subgroup of obese patients without MS $(n=18$; NAFLD $-5.9 \pm 2.4$ vs NAFLD $+8 \pm 2.4 \mathrm{pmol} /$ $\mathrm{L}, p=0.06 ; \mathrm{NASH}-6.5 \pm 2.6$ vs $\mathrm{NASH}+8.5 \pm 0.2 \mathrm{pmol} / \mathrm{L}$, $p=0.01$ ). Furthermore, the association between higher copeptin levels and NAFLD persisted significantly when assessed in the partial correlation analysis adjusted for the presence of MS (correlation's coefficient $=0.32, p=0.017$ ).

The presence of NAFLD correlated with higher copeptin levels and, as expected, with all the clinical parameters associated with MS, such as greater BMI, waist circumference, FBG, presence of T2DM, and atherogenic dyslipidemia, whereas no association was found between NAFLD, sex, and age (Additional file 1: Table S1).
In obese individuals, circulating copeptin levels positively correlated with the percentage of macro- and micro-vesicular steatosis, lobular inflammation, the NAS score for diagnosis of NASH, and the SAF score for fibrosis (Table 2). Furthermore, copeptin significantly increased throughout degrees of NASH severity, as expressed as absence $(0, n=14)$, borderline $(1, n=5)$, and overt $(2, n=13) \mathrm{NASH}$ at the liver biopsy (Fig. 1).

Moreover, higher copeptin was associated with male sex and greater serum uric acid and creatinine concentration in all the study participants (Table 2 and Additional file 1: Table S2). In the entire study population, plasma copeptin concentration positively correlated also with greater waist

Table 2 Copeptin-bivariate correlation analyses (Pearson's coefficient, *Spearman's coefficient, copeptin is considered as a continuous variable). Cohort obese individuals $(n=60)$

\begin{tabular}{|c|c|c|}
\hline & Correlation coefficient & $p$ value \\
\hline Age & 0.048 & 0.75 \\
\hline $\operatorname{Sex}(M / F)$ & -0.44 & $0.001^{*}$ \\
\hline $\mathrm{BMI}$ & 0.03 & 0.84 \\
\hline Waist circumference & 0.03 & 0.87 \\
\hline $\mathrm{SBP}(\mathrm{mmHg})$ & -0.046 & 0.76 \\
\hline $\mathrm{DBP}$ & -0.040 & 0.80 \\
\hline FBG & -0.25 & 0.08 \\
\hline Total cholesterol & -0.06 & 0.71 \\
\hline $\mathrm{HDL}$ & -0.07 & 0.65 \\
\hline LDL & 0.19 & 0.23 \\
\hline Triglycerides & -0.21 & 0.18 \\
\hline AST & 0.13 & 0.37 \\
\hline $\mathrm{ALT}$ & 0.27 & 0.12 \\
\hline Serum creatinine & 0.31 & 0.045 \\
\hline Serum uric acid & 0.33 & 0.045 \\
\hline НOMА- $\beta \%$ & 0.05 & 0.76 \\
\hline HOMA-IR & 0.07 & 0.70 \\
\hline T2DM yes/no & -0.20 & $0.15^{*}$ \\
\hline MS yes/no & -0.16 & $0.29^{*}$ \\
\hline NAFL & 0.31 & $0.015^{*}$ \\
\hline $\mathrm{NASH}$ & 0.40 & $0.002^{*}$ \\
\hline NAS: macro-vesicular steatosis & 0.36 & $0.026^{*}$ \\
\hline NAS: micro-vesicular steatosis & 0.31 & $0.05^{*}$ \\
\hline NAS: lobular inflammation & 0.37 & $0.024^{*}$ \\
\hline NAS score & 0.35 & $0.03^{*}$ \\
\hline SAF score & 0.44 & $0.001^{*}$ \\
\hline
\end{tabular}

Abbreviations: SBP systolic blood pressure, DBP diastolic blood pressure, $H D L-C$ high-density lipoprotein cholesterol, $L D L-C$ low-density lipoprotein, $A S T$ aspartate aminotransferase, $A L T$ alanine aminotransferase, $F B G$ fasting blood glucose, $F B$ I fasting blood insulin, HOMA-IR HOmeostasis Model Assessment of insulin resistance, HOMA- $\beta \%$ HOmeostasis Model Assessment of insulin secretion, MS metabolic syndrome, T2DM type 2 diabetes mellitus, NAS NAFLD activity score, SAF steatosis, activity, and fibrosis 


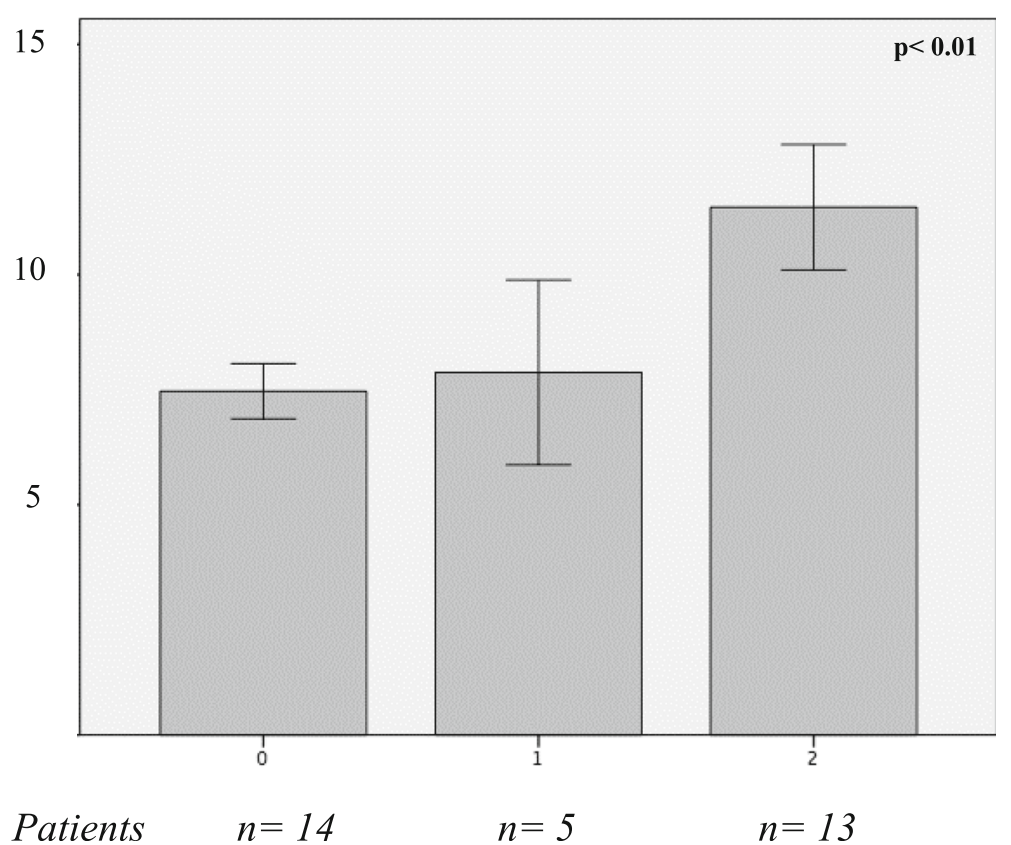

Fig. 1 Plasma copeptin (pmol/L) in relation to the presence and severity of NASH $(0=$ absent; $1=$ borderline; $2=$ NASH). ANOVA test applied

circumference and with a higher prevalence of MS and NAFLD (Additional file 1: Table S2).

When comparing clinical characteristics of study participants in relation to the circulating copeptin levels, we observed that patients belonging to the highest copeptin quartile had a higher prevalence of male sex, MS, NAFLD and NASH and greater waist circumference, serum creatinine, and uric acid than those belonging to the lowest quartile (Table 3). Furthermore, higher copeptin concentration predicted the presence of NASH at the liver biopsy in the ROC curve with AUROC $=0.822(95 \% \mathrm{CI}$ $0.71-0.93, p<0.001$, Additional file 1: Figure S1).

Finally, in a logistic regression model adjusted for age, sex, renal function, presence of T2DM, and MS components, copeptin levels predicted the presence of NASH at the liver biopsy (Table 4). The association between copeptin and NASH persisted statistically significant also after further adjustment for each individual metabolic parameter (BMI, FBG, triglycerides and HDL-c), entered as continuous variables in progressive conditional forward regression models $($ copeptin-standardized $\beta$-coefficient $=$ $0.64, p=0.035$, odds ratio $=1.97,95 \% \mathrm{CI}=1.05-3.69$; Cox and Snell $R^{2}=0.56$; Additional file 1: Table S3).

\section{Discussion}

In this study, we demonstrated for the first time in humans the existence of a strong association between elevated circulating copeptin levels and the presence of NAFLD and NASH. Higher plasma copeptin was associated with greater intrahepatic fat content and inflammation at the liver histology as well as increasing severity of NASH.

We also show an association between copeptin, visceral obesity, and the presence of MS, a finding in line with previous studies demonstrating a role of copeptin in glucose and lipid metabolism $[3,4,6,17,34]$ which likely underlies the increased risk of metabolic and cardiovascular diseases associated to elevated copeptin observed in many longitudinal studies [7-16].

The predictive value of copeptin in identifying NAFLD and NASH in obese individuals was independent from other dysmetabolic conditions, such as increased body adiposity and the diagnosis of T2DM and MS, even if an association between higher copeptin and metabolic impairment was also observed. In our population of obese individuals, the prevalence of MS reached $70 \%$ whereas T2DM was diagnosed in 14\% and biopsy-proven NAFLD in just over $50 \%$ participants. Therefore, we may speculate that, according to the metabolic profiling, at least one third of these patients had not (yet) clinically relevant metabolic impairment but obesity, at the time of study recruitment, thus explaining the observation of some overlap in few parameters, such as fasting insulin and its derived indexes, between obese and non-obese subjects.

In line with previous investigations, we observed an association between male sex and greater copeptin concentration. In order to exclude the possible interference of sex distribution behind the association between copeptin and NAFLD, we first explored the association between NAFLD and sex, finding no relationship 
Table 3 Clinical characteristics to study participants $(n=120)$ in relation to the quartile of plasma copeptin concentration

\begin{tabular}{|c|c|c|c|c|c|}
\hline & $\begin{array}{l}\text { Copeptin } \\
\text { quartile } 1\end{array}$ & $\begin{array}{l}\text { Copeptin } \\
\text { quartile } 2\end{array}$ & $\begin{array}{l}\text { Copeptin } \\
\text { quartile } 3\end{array}$ & $\begin{array}{l}\text { Copeptin } \\
\text { quartile } 4\end{array}$ & $p$ value \\
\hline Age (years) & $43.8 \pm 9.2$ & $46.2 \pm 10.7$ & $43.6 \pm 12$ & $45.7 \pm 10.1$ & 0.48 \\
\hline $\operatorname{Sex}(\% \mathrm{M})$ & $50 \%$ & $30 \%$ & $30 \%$ & $90 \%$ & $<0.001^{*} ; 0.01 \wedge$ \\
\hline $\mathrm{BMI}\left(\mathrm{kg} / \mathrm{m}^{2}\right)$ & $32.6 \pm 10.9$ & $32.3 \pm 9.8$ & $36.9 \pm 10.8$ & $34.7 \pm 11.2$ & 0.51 \\
\hline Waist circumference $(\mathrm{cm})$ & $92.7 \pm 19.3$ & $100.5 \pm 27.4$ & $102.2 \pm 27.12$ & $113.4 \pm 22.8$ & 0.013 \\
\hline PAS (mmHg) & $115 \pm 29.4$ & $125.4 \pm 14.4$ & $120.7 \pm 13$ & $124.4 \pm 14.5$ & 0.16 \\
\hline PAD (mmHg) & $77.1 \pm 6.3$ & $85.5 \pm 21.7$ & $81.6 \pm 9.25$ & $80.5 \pm 10.6$ & 0.17 \\
\hline FBG $(\mathrm{mg} / \mathrm{dL})$ & $91(86.5-94.5)$ & $94.5(84.5-122)$ & $94(86.5-103.5)$ & $95(85.3-100.5)$ & 0.56 \\
\hline $\mathrm{FBI}(\mu \mathrm{U} \cup / \mathrm{mL})$ & $11.1(10.6-20.3)$ & $12.2(8.7-25.2)$ & $12.1(8.8-27.4)$ & $11.3(8.8-17.2)$ & 0.96 \\
\hline HOMA-IR & $3.3(2.4-4.02)$ & $3.6(1.9-5.6)$ & $2.8(1.97-5.9)$ & $2.73(1.8-4.3)$ & 0.60 \\
\hline HOMA- $\beta \%$ & $151.7(122.2-496.1)$ & $89.6(63.8-121)$ & $163.5(107.9-274)$ & $94(133.5-199.7)$ & 0.056 \\
\hline AST (IU/L) & $20(15-22.3)$ & $20(16-23)$ & $23(17.5-29)$ & $18(15.3-25.3)$ & 0.62 \\
\hline ALT (IU/L) & $21(15.7-24.3)$ & $18(15-28)$ & $25(16.5-48)$ & $18(15-36.5)$ & 0.36 \\
\hline GGT (IU/L) & $16(12.5-20)$ & $13(11-18.8)$ & $25(16.5-48)$ & $18(10.8-22.5)$ & 0.53 \\
\hline Total cholesterol (mg/dL) & $200.6 \pm 49.6$ & $205.9 \pm 36$ & $180.9 \pm 27$ & $204.5 \pm 30.5$ & 0.75 \\
\hline $\mathrm{HDL}(\mathrm{mg} / \mathrm{dL})$ & $55.4 \pm 13.6$ & $56.1 \pm 14.4$ & $43.6 \pm 7.8$ & $52.4 \pm 11.5$ & 0.44 \\
\hline LDL (mg/dL) & $115.1 \pm 35.2$ & $123.3 \pm 34$ & $111.7 \pm 25.4$ & $122.7 \pm 37.4$ & 0.51 \\
\hline Triglycerides (mg/dL) & $83(72-139)$ & $127(73-164)$ & $118.5(87-146.5)$ & $102.5(73.5-150.3)$ & 0.43 \\
\hline Serum creatinine $(\mathrm{mg} / \mathrm{mL})$ & $0.68 \pm 0.09$ & $0.8 \pm 0.15$ & $0.74 \pm 0.013$ & $0.8 \pm 0.17$ & 0.01 \\
\hline Serum uric acid (mg/mL) & $4.8 \pm 1.02$ & $5.5 \pm 1.4$ & $5.12 \pm 1.1$ & $6.1 \pm 1.6$ & 0.037 \\
\hline NAFLD (\%) & $15 \%$ & $28 \%$ & $40 \%$ & $42 \%$ & $0.026^{*} ; 0.09 \wedge$ \\
\hline Biopsy-proven NASH & $10 \%$ & $0 \%$ & $40 \%$ & $50 \%$ & $0.05^{*} ; 0.004 \wedge$ \\
\hline T2DM (\%) & $7 \%$ & $15 \%$ & $12 \%$ & $0 \%$ & $0.18^{*} ; 0.16 \wedge$ \\
\hline MS (\%) & $26 \%$ & $26 \%$ & $48 \%$ & $52 \%$ & $0.051^{*} ; 0.22 \wedge$ \\
\hline
\end{tabular}

${ }^{*}$ Chi-square test. $p$ values related to the comparison between copeptin quartiles 1 vs $4 . \wedge p$ value related to distribution across all the quartiles (Kruskal-Wallis test) Abbreviations: BMI body mass index, SBP systolic blood pressure, DBP diastolic blood pressure, $H D L-C$ high-density lipoprotein cholesterol, $L D L-C$ low-density lipoprotein, AST aspartate aminotransferase, ALT alanine aminotransferase, GGT gamma-glutamyl transpeptidase, FBG fasting blood glucose, FBI fasting blood insulin, HOMA-IR HOmeostasis Model Assessment of insulin resistance, HOMA- $\beta \%$ HOmeostasis Model Assessment of insulin secretion, NAFLD non-alcoholic fatty liver disease, NASH non-alcoholic steatohepatitis, MS metabolic syndrome, T2DM type 2 diabetes mellitus

Table 4 Multivariate logistic regression analysis. The presence of NASH is the dependent variable. Copeptin is considered as a continuous variable

\begin{tabular}{|c|c|c|c|c|c|c|c|c|}
\hline & \multirow[t]{2}{*}{$\beta$} & \multirow[t]{2}{*}{ S.E. } & \multirow[t]{2}{*}{ Wald } & \multirow{2}{*}{$\begin{array}{l}\beta- \\
\text { standardized }\end{array}$} & \multirow{2}{*}{$\begin{array}{l}p \\
\text { value }\end{array}$} & \multirow{2}{*}{$\begin{array}{l}\text { Odds } \\
\text { ratio }\end{array}$} & \multicolumn{2}{|l|}{$95 \% \mathrm{Cl}$} \\
\hline & & & & & & & Lower & Upper \\
\hline Age & 0.33 & 0.17 & 3.47 & 0.63 & 0.06 & 1.39 & 0.98 & 1.95 \\
\hline $\operatorname{Sex}(M / F)$ & -1.08 & 1.88 & 0.33 & -0.11 & 0.57 & 0.34 & 0.008 & 13.57 \\
\hline Copeptin & 0.547 & 0.27 & 4.09 & 0.54 & 0.043 & 1.73 & 1.02 & 2.93 \\
\hline T2DM (yes/no) & 1.65 & 1.85 & 0.79 & 0.13 & 0.37 & 5.2 & 0.14 & 197.5 \\
\hline Serum creatinine & 0.57 & 5.4 & 0.01 & -0.02 & 0.92 & 1.77 & 0.000 & 7420 \\
\hline Dyslipidemia (yes/no) & 0.43 & 2.80 & 2.41 & 0.03 & 0.12 & 0.013 & 0.000 & 3.12 \\
\hline Number of MS components & 3.46 & 1.71 & 4.09 & 0.36 & 0.043 & 31.8 & 1.11 & 909.1 \\
\hline
\end{tabular}

Cox and Snell $R^{2}=0.408$

Abbreviations: S.E. standard error, $\mathrm{Cl}$ confidence interval, T2DM type 2 diabetes mellitus, MS metabolic syndrome 
between these two variables. Thereafter, we built several sex- and age-forced multivariate models confirming that sex did not represent a confounder in the association between NAFLD and higher copeptin levels. Furthermore, in our study, plasma copeptin and histological parameters associated with NASH correlated in a dosedependent manner, which may represent a direct action of VP on liver parenchyma.

The only previous investigation on VP and liver steatosis has been conducted on animal models by Taveau and collaborators [17], who explored the impact of experimentally induced high and low circulating VP levels on glucose homeostasis in obese rats. The authors demonstrated impaired glucose regulation in the presence of high VP levels and a surprisingly reduced hepatic fat content after lowering circulating VP concentration by exposing obese rats to 2-week high water intake. As compared with control obese rats, water-induced low VP reduced the intrahepatic lipid content and the expression of proteins involved in lipogenesis whereas it increased the hepatic glycogen content [17]. Intriguingly, the authors observed an unexpected dissociation between the effects of decreased VP levels on glucose homeostasis and hepatic fat accumulation: low-VP rats did not display a significantly improved glucose tolerance even though they exhibited a marked decrease in hepatic steatosis, whereas rats treated with VP infusion in the same study exhibited impaired glucose tolerance but no aggravation of hepatic steatosis [17].

Liver is a well-known target of VP action, and the $\mathrm{V} 1 \mathrm{aR}$ is widely expressed in hepatic tissue, where it mainly regulates lipid metabolism promoting lipogenesis $[23,35]$ and stimulating bile acid production [23]; accordingly, V1aR knock-out mice have low blood triglycerides and increased ketone bodies when compared to wild type, confirming the antilipolytic action of VP [23]. $\mathrm{VP} / \mathrm{V} 1 \mathrm{aR}$ system has been widely studied in relation to the progression to cirrhosis and hepatic decompensation of several acute and chronic liver diseases [24, 25, 3638]. Indeed, the secretion of VP is involved in advanced stages of chronic liver diseases; VP counteracts the reduction of the arterial pressure induced by splanchnic vasodilatation and subsequent increased peripheral vascular resistance. In this scenario, although the non-osmotic secretion of VP may temporarily preserve the arterial blood volume, it is associated with the development of ascites, hepato-renal syndrome, and detrimental clinical outcomes. Thus, Solà and collaborators [24] first demonstrated that plasma copeptin represents a prognostic factor for disease progression, clinical decompensation, and prognosis in patients with cirrhosis [24], and these findings were confirmed in other investigations [36-38]. In our study, no participant had any clinical or biochemical signs of hepatic damage, making it unlikely that mechanisms involving fluid distribution and vascular compliance underlie the association between high copeptin and NAFLD in our study.

The cross-sectional design of our study does not allow us to establish a causal nexus between these findings; however, it is plausible to hypothesize that increased VP, as measured by plasma copeptin levels, play a direct role in inducing intrahepatic fat accumulation by modulating lipid metabolism towards antilipolysis. The VP system has been hypothesized to represent a unifying factor behind the MS and a link between metabolic and cardiovascular disease $[9,16]$. As liver steatosis is considered as a feature of the MS, our findings add further knowledge on metabolic complications associated with an impaired VP/V1aR system and provide novel insights on possible mechanisms underlying NAFLD and NASH.

\section{Conclusion}

Our study demonstrates for the first time that copeptin levels predict the presence of biopsy-proven NAFLD in obese individuals, regardless of body adiposity and the coexistence of other metabolic disorders. Studies with longitudinal design are warranted for exploring the possible involvement of AVP/V1aR system in the development and progression of NAFLD.

\section{Additional file}

Additional file 1: Table S1. Presence of NAFLD - Bivariate correlation analyses. Spearman's coefficient, NAFLD is considered as a dichotomous variable (Yes/No). Table S2. Copeptin- Bivariate correlation analyses (Pearson's coefficient, *Spearman's coefficient, copeptin is considered as a continuous variable). Table S3. Multivariate logistic regression analysis. The presence of NASH is the dependent variable. Copeptin is considered as a continuous variable. Figure S1. Copeptin area under the receiver operating characteristic (ROC) curve for NASH. (DOCX $77 \mathrm{~kb}$ )

\section{Acknowledgements}

Not applicable.

\section{Funding}

This study was funded by grants from Sapienza University. Prof. Melander was supported by grants from Knut and Alice Wallenberg Foundation, Göran Gustafsson Foundation, the Swedish Heart- and Lung Foundation, the Swedish Research Council, the Novo Nordisk Foundation, Region Skåne, Skåne University Hospital and the Swedish Foundation for Strategic Research Dnr IRC15-0067.

\section{Availability of data and materials}

All data generated or analyzed during this study are included in this published article and its supplementary information files (Additional file 1).

\section{Authors' contributions}

IB, OM, SE, and MGC conceived the study. IB, MGC, FL, DC, and FAC coordinated the study, oversaw patient recruitment, and finalized the data set. FAC, IB, and DC oversaw collection and analysis of biological samples. IB, MCG, and OM performed statistical analyses. GS performed all the liver biopsies. CDC and CC read all the biopsies, performed the experiments, and finalized the data set. IB, SE, OM, and MGC drafted the paper, which was reviewed by all authors. All authors read and approved the final manuscript. 


\section{Ethics approval and consent to participate}

The study protocol was reviewed and approved by the local Ethics Committee at the Sapienza University of Rome, Italy, and conducted in conformance with the Helsinki Declaration. Informed written consent was obtained from all patients before all the study procedures.

\section{Consent for publication}

Not applicable.

\section{Competing interests}

The authors declare that they have no competing interests.

\section{Publisher's Note}

Springer Nature remains neutral with regard to jurisdictional claims in published maps and institutional affiliations.

\section{Author details}

${ }^{1}$ Department of Experimental Medicine, Policlinico Umberto I, Sapienza University of Rome, Rome, Italy. ${ }^{2}$ Department of Clinical Sciences, Lund University, Malmoe, Sweden. ${ }^{3}$ Department of Medical-Surgical Sciences and Biotechnologies, Sapienza University of Rome, Rome, Italy.

\section{Received: 30 November 2018 Accepted: 9 April 2019}

\section{Published online: 30 April 2019}

\section{References}

1. Struck J, Morgenthaler NG, Bergmann A. Copeptin, a stable peptide derived from the vasopressin precursor, is elevated in serum of sepsis patients. Peptides. 2005. https://doi.org/10.1016/j.peptides.2005.04.019.

2. Roussel R, Fezeu L, Marre M, Velho G, Fumeron F, Jungers $P$, et al. Comparison between copeptin and vasopressin in a population from the community and in people with chronic kidney disease. J Clin Endocrinol Metab. 2014. https://doi.org/10.1210/jc.2014-2295.

3. Keppens $\mathrm{S}$, de Wulf $\mathrm{H}$. The nature of the hepatic receptors involved in vasopressin-induced glycogenolysis. Biochim Biophys Acta. 1979;588(1):63-9.

4. Whitton PD, Rodrigues LM, Hems DA. Stimulation by vasopressin, angiotensin and oxytocin of gluconeogenesis in hepatocyte suspensions. Biochem J. 1978;176(3):893-8.

5. Pollard AD, Brindley DN. Effects of vasopressin and corticosterone on fatty acid metabolism and on the activities of glycerol phosphate acyltransferase and phosphatidate phosphohydrolase in rat hepatocytes. Biochem J. 1984; 217(2):461-9.

6. Abu-Basha EA, Yibchok-Anun S, Hsu WH. Glucose dependency of arginine vasopress-induced insulin and glucagon release from the perfused rat pancreas. Metabolism. 2002;51(9):1184-90.

7. Enhörning S, Wang TJ, Nilsson PM, Almgren P, Hedblad B, Berglund G, et al. Plasma copeptin and the risk of diabetes mellitus. Circulation. 2010;121(19): 2102-8.

8. Enhörning S, Bankir L, Bouby N, Struck J, Hedblad B, Persson M, et al. Copeptin, a marker of vasopressin, in abdominal obesity, diabetes and microalbuminuria: the prospective Malmö Diet and Cancer Study cardiovascular cohort. Int J Obes. 2013. https://doi.org/10.1038/ijo.2012.88.

9. Enhörning S, Hedblad B, Nilsson PM, Engström G, Melander O. Copeptin is an independent predictor of diabetic heart disease and death. Am Heart $J$. 2015. https://doi.org/10.1016/j.ahj.2014.11.020.

10. Riphagen IJ, Boertien WE, Alkhalaf A, Kleefstra N, Gansevoort RT, Groenier KH, et al. Copeptin, a surrogate marker for arginine vasopressin, is associated with cardiovascular and all-cause mortality in patients with type 2 diabetes (ZODIAC-31). Diabetes Care. 2013. https://doi.org/10.2337/dc12-2165.

11. Tasevska I, Enhörning S, Persson M, Nilsson PM, Melander O. Copeptin predicts coronary artery disease cardiovascular and total mortality. Heart. 2016. https://doi.org/10.1136/heartjnl-2015-308183.

12. Melander O. Vasopressin, from regulator to disease predictor for diabetes and cardiometabolic risk. Ann Nutr Metab. 2016. https://doi.org/10.1159/ 000446201.

13. Abbasi A, Corpeleijn E, Meijer E, Postmus D, Gansevoort RT, Gans RO, et al. Sex differences in the association between plasma copeptin and incident type 2 diabetes: the prevention of renal and vascular endstage disease (PREVEND) study. Diabetologia. 2012. https://doi.org/10.1007/s00125-012-2545-x.
14. Wannamethee SG, Welsh P, Papacosta O, Lennon L, Whincup PH, Sattar N Copeptin, insulin resistance, and risk of incident diabetes in older men. J Clin Endocrinol Metab. 2015. https://doi.org/10.1210/JC.2015-2362.

15. Saleem U, Khaleghi M, Morgenthaler NG, Bergmann A, Struck J, Mosley TH $\mathrm{Jr}$, et al. Plasma carboxy-terminal provasopressin (copeptin): a novel marker of insulin resistance and metabolic syndrome. J Clin Endocrinol Metab. 2009. https://doi.org/10.1210/jc.2008-2278.

16. Enhörning S, Struck J, Wirfält E, Hedblad B, Morgenthaler NG, Melander O. Plasma copeptin, a unifying factor behind the metabolic syndrome. J Clin Endocrinol Metab. 2011. https://doi.org/10.1210/jc.2010-2981.

17. Taveau C, Chollet C, Waeckel L, Desposito D, Bichet DG, Arthus MF, et al. Vasopressin and hydration play a major role in the development of glucose intolerance and hepatic steatosis in obese rats. Diabetologia. 2015. https:// doi.org/10.1007/s00125-015-3496-9.

18. Clark JM, Brancati FL, Diehl AME. Nonalcoholic fatty liver disease: the most common cause of abnormal liver enzymes in the US population. Gastroenterology. 2011;120(5 Suppl 1):A65.

19. Browning JD, Szczepaniak LS, Dobbins R, Nuremberg P, Horton JD, Cohen JC, Grundy SM, Hobbs HH. Prevalence of hepatic steatosis in an urban population in the United States: impact of ethnicity. Hepatology. 2004;40:1387-95.

20. Bedogni G, Miglioli L, Masutti F, Castiglione A, Crocè LS, Tiribelli C, Bellentani $S$. Incidence and natural course of fatty liver in the general population: the Dionysos study. Hepatology. 2007;46:1387-91.

21. Targher G, Day CP, Bonora E. Risk of cardiovascular disease in patients with nonalcoholic fatty liver disease. N Engl J Med. 2010. https://doi.org/10.1056/ NEJMra0912063.

22. Brodosi L, Marchignoli F, Petroni ML, Marchesini GNASH. A glance at the landscape of pharmacological treatment. Ann Hepatol. 2016. https://doi.org/ 10.5604/16652681.1212318

23. Hiroyama M, Aoyagi T, Fujiwara Y, Birumachi J, Shigematsu Y, Kiwaki K, et al. Hypermetabolism of fat in V1a vasopressin receptor knockout mice. Mol Endocrinol. 2007;21(1):247-58.

24. Solà E, Kerbert AJ, Verspaget HW, Moreira R, Pose E, Ruiz P, et al. Plasma copeptin as biomarker of disease progression and prognosis in cirrhosis. J Hepatol. 2016. https://doi.org/10.1016/j.jhep.2016.07.003.

25. Tawfik AK, Helmy A, Yousef M, Abou-Saif S, Kobtan A, Asaad E, Abd-Elsalam S. Copeptin as a novel marker predicting prognosis of liver cirrhosis and its major complications. Hepat Med. 2018. https://doi.org/10.2147/HMER.S174267.

26. Grundy SM, Cleeman JI, Daniels SR, Donato KA, Eckel RH, Franklin BA, et al. Diagnosis and management of the metabolic syndrome. An American Heart Association/National Heart, Lung, and Blood Institute Scientific Statement. Executive Summary. Circulation. 2005;112:e285-90.

27. American Diabetes Association. Standards of medical care in diabetes-2009. Diabetes Care. 2009:32:S13-61.

28. Saverymuttu SH, Joseph AE, Maxwell JD. Ultrasound scanning in the detection of hepatic fibrosis and steatosis. Br Med J (Clin Res Ed). 1986; 292(6512):13-5.

29. Matsuda M, DeFronzo RA. Insulin sensitivity indices obtained from oral glucose tolerance testing: comparison with the euglycemic insulin clamp. Diabetes Care. 1999;22:1462-70.

30. Rockey DC, Caldwell SH, Goodman ZD, Nelson RC, Smith AD, American Association for the Study of Liver Diseases. Liver biopsy. Hepatology. 2009; 49(3):1017-44

31. Colloredo G, Guido M, Sonzogni A, Leandro G. Impact of liver biopsy size on histological evaluation of chronic viral hepatitis: the smaller the sample, the milder the disease. J Hepatol. 2003;39:239-44.

32. Brunt EM, Janney CG, Di Bisceglie AM, Neuschwander-Tetri BA, Bacon BR. Nonalcoholic steatohepatitis: a proposal for grading and staging the histological lesions. Am J Gastroenterol. 1999;94:2467-74.

33. Kleiner DE, Brunt EM, Van Natta M, Behling C, Contos MJ, Cummings OW et al. Nonalcoholic Steatohepatitis Clinical Research Network. Design and validation of a histological scoring system for nonalcoholic fatty liver disease. Hepatology 2005; 41:1313-1321.

34. Aoyagi T, Birumachi J, Hiroyama M, Fujiwara Y, Sanbe A, Yamauchi J, et al. Alteration of glucose homeostasis in V1a vasopressin receptor-deficient mice. Endocrinology. 2007;148(5):2075-84.

35. Nakamura K, Velho G, Bouby N. Vasopressin and metabolic disorders: translation from experimental models to clinical use. J Intern Med. 2017. https://doi.org/10.1111/joim.12649.

36. Kerbert AJ, Verbeke L, Chiang FW, Laleman W, van der Reijden JJ, van Duijn W, et al. Copeptin as an indicator of hemodynamic derangement and 
prognosis in liver cirrhosis. PLoS One. 2015. https://doi.org/10.1371/journal. pone.0138264.

37. Kerbert AJC, Verspaget HW, Navarro ÀA, Jalan R, Solà E, Benten D, et al. Copeptin in acute decompensation of liver cirrhosis: relationship with acute-on-chronic liver failure and short-term survival. Crit Care. 2017;21 (1): 321. https://doi.org/10.1186/s13054-017-1894-8.

38. Moreno JP, Grandclement E, Monnet E, Clerc B, Agin A, Cervoni JP, et al. Plasma copeptin, a possible prognostic marker in cirrhosis. Liver Int. 2013. https://doi.org/10.1111/liv.12175.

Ready to submit your research? Choose BMC and benefit from:

- fast, convenient online submission

- thorough peer review by experienced researchers in your field

- rapid publication on acceptance

- support for research data, including large and complex data types

- gold Open Access which fosters wider collaboration and increased citations

- maximum visibility for your research: over $100 \mathrm{M}$ website views per year

At $\mathrm{BMC}$, research is always in progress.

Learn more biomedcentral.com/submissions 